\title{
Globe
}

Revue internationale d'études québécoises

\section{Louise Vigneault : Identité et modernité dans l'art au Québec. Borduas, Sullivan, Riopelle. Montréal, Les Cahiers du Québec, 2002}

\section{Julie Lavigne}

Volume 6, numéro 2, 2003

URI : https://id.erudit.org/iderudit/1000826ar

DOI : https://doi.org/10.7202/1000826ar

Aller au sommaire du numéro

Éditeur(s)

Globe, Revue internationale d'études québécoises

ISSN

1481-5869 (imprimé)

1923-8231 (numérique)

Découvrir la revue

Citer ce compte rendu

Lavigne, J. (2003). Compte rendu de [Louise Vigneault : Identité et modernité dans l'art au Québec. Borduas, Sullivan, Riopelle. Montréal, Les Cahiers du Québec, 2002]. Globe, 6(2), 192-195. https://doi.org/10.7202/1000826ar d'utilisation que vous pouvez consulter en ligne.

https://apropos.erudit.org/fr/usagers/politique-dutilisation/ 
moins connues. Il aurait été opportun d'effectuer une démarche relevant de la gynocritique afin d'identifier davantage ces voix " mineures " que l'institution littéraire a écartées. En dépit de ces omissions, l'ouvrage parvient à rendre compte de tout un pan de l'évolution de la société québécoise.

Lucie Guillemette et Cynthia Lévesque Université du Québec à Trois-Rivières

\section{Louise Vigneault}

Identité et modernité dans l'art au Québec.

Borduas, Sullivan, Riopelle.

Montréal, Les Cahiers du Québec, 2002.

La question de l'identité au Québec a fait l'objet de nombreuses publications en sciences sociales depuis quelques années déjà. Par contre, rares sont les recherches qui s'interrogent sur la thématique de l'identité québécoise dans l'art. Louise Vigneault tente donc, par cet ouvrage, de combler cette absence en étudiant trois figures de proue de l'art québécois : Paul-Émile Borduas, Françoise Sullivan et Jean-Paul Riopelle.

Dès sa brève introduction, Louise Vigneault présente une approche multidisciplinaire remarquable; l'histoire de l'art se voit enrichie par des emprunts aux principales sciences sociales: l'histoire, l'anthropologie, l'ethnologie, la sociologie, les sciences politiques et la philosophie. C'est en fonction de cette perspective large que l'auteure définit le concept d'identité du sujet moderne principalement dans sa relation à l'Autre. D'abord centrée sur le sujet moderne en général, sa définition de l'identité glisse rapidement vers le contexte québécois. L'identité canadiennefrançaise y est décrite comme une identité collective aliénée par une non-reconnaissance de l'Autre et placée dans une position de méfiance et de survie. Vigneault situe donc les trois artistes dans ce contexte identitaire particulier ; plus précisément, l'auteure relève le dilemme des artistes entre une position moderniste en art - qui tend vers l'autoréférentialité du médium artistique - et l'affirmation d'une identité nationale aux prises avec un mandat de survivance du caractère canadien-français.

Vigneault dresse dans le premier chapitre un portrait socio-politique et historique du milieu artistique depuis le début du siècle dernier jus- 
qu'aux débuts de Borduas. Elle dégage les principales stratégies artistiques de survivance de l'identité canadienne-française dans les œuvres de Clarence Gagnon, de Marc-Aurèle Fortin, d'Alfred Laliberté et de MarcAurèle de Foy Suzor-Coté. Ainsi, plutôt que de suivre le mouvement significatif de modernisation que connaît le Québec en ce début de siècle, le milieu des arts visuels adopte une attitude nostalgique avec des thématiques issues du siècle dernier. Les thèmes idéalisés de la ruralité et du terroir canadien-français sont donc dominants chez les artistes de cette génération et sont accompagnés d'une valorisation du folklore national. Conséquemment, le retour au folklore a pour effet de promouvoir et d'objectiver la culture canadienne-française. Cette objectivation aurait permis de fonder des référents identitaires clairs, nécessaires alors pour faire face à l'instabilité culturelle nationale.

C'est dans ce contexte artistique conservateur que Borduas tente, selon Vigneault, d'imposer sa peinture moderniste et sa conception d'un sujet affranchi et moderne. Cependant, la quête de Borduas fut complexe. En effet, bien qu'il tente un rattrapage artistique visant un positionnement dans l'avant-garde artistique internationale, il désire néanmoins marquer une position identitaire " authentique ". La stratégie que choisit Borduas est celle du primitivisme, laquelle s'avère aussi bien artistique qu'identitaire, mais en phase avec l'actualité internationale et résolument moderne: "De concert avec la perception moderne de l'identité, le primitivisme entretient la croyance selon laquelle il existe un soi "véritable", authentique, à reconquérir. "(p. 119). Dans le contexte québécois, le primitivisme revêt une importance particulière, car il permet d'élargir les modèles identitaires et de " redéfinir l'identité collective du Québec moderne " (p. 121) sans renvoyer à la mère patrie ni souscrire à l'idéologie clérico-nationaliste. À la lumière des écrits de Borduas, du Refus global, de sa correspondance et des titres de ses œuvres, Vigneault retrace les différents modèles identificatoires de l'artiste. Le primitivisme chez Borduas a pour principal relais l'enfant créateur et spontané, non entaché par la société opprimante, mais emprunte aussi les figures du créateur autodidacte, de l'art populaire, de l'île comme lieu authentique à l'écart de la culture, et enfin, celle de l'autochtone. À partir de ces différentes identifications, de ses écrits et, dans une moindre mesure, de sa pratique artistique, l'auteure affirme que le positionnement identitaire de Borduas oscille entre deux pôles mythiques. D'un côté, à l'image des Patriotes, il s'incarne dans le rebelle révolutionnaire cherchant à libérer le peuple et l'art; de l'autre, il se confond avec l'image du "martyr 
sacrifié ", notamment à cause du scandale lié à la publication du manifeste Refus global et de la non-reconnaissance de sa production artistique au Québec avant sa mort.

Pour aborder la figure de Françoise Sullivan, Vigneault fait principalement appel à une histoire féministe de l'art proche de celle pratiquée par Griselda Pollock et par Linda Nochlin. Le fait féminin de l'artiste est donc une donnée d'importance. Selon l'auteure, Sullivan opte elle aussi pour la stratégie artistique du primitivisme. Ici, ce "détour par l'Autre * qu'est le primitivisme a un écho tout particulier. En effet, comme les féministes post-colonialistes l'ont fait remarquer, les traits associés aux femmes sont sensiblement les mêmes que ceux attribués au primitif : le sceau de l'Autre, la différence, "le sujet incomplet ". Dans sa production picturale, le primitivisme de Sullivan passe principalement par une identification à l'autochtone. Cette identification lui permet non seulement de se distancier du modèle colonial français, mais aussi du modèle canadien-français, occidental et masculin. En passant par la figure de l'autochtone, Sullivan n'affiche pas exclusivement sa marginalité sexuelle, ce qui lui permet, selon l'auteure, d' "éviter l'essentialisme féminin *. De cette manière, l'identification n'est plus subie mais affirmée et devient alors productive. Dans sa danse, paradoxalement, Sullivan s'inspire du modèle essentialiste de la "femme archaiqque ", c'est-à-dire l'archétype de la femme issue d'une époque pré-patriarcale (paradoxe que l'auteure ne souligne pas). Par cette voie, l'artiste cherche à " magnifier le pouvoir créatif féminin - et à imposer une image positive et active de la femme. C'est à New York, auprès de Franzisca Boas, que Sullivan fait son apprentissage, dans un style empruntant à la fois à l'expressionnisme européen, aux nouvelles tendances américaines et aux pratiques non-occidentales (notamment africaines et amérindiennes). Par cette danse multiethnique, Sullivan s'identifie encore à l'Autre et se positionne en marge de la "xénophobie inculquée par l'idéologie clériconationaliste "; mieux encore, elle la subvertit. De plus, elle critique par le choix de son médium artistique cette même idéologie qui voit dans la danse une activité reliée à l'Autre et au mal.

D'abord réfractaire à l'abstraction, Riopelle dépasse rapidement Borduas, selon Vigneault, dans la quête avant-gardiste de l'autoréférentialité picturale. Riopelle quitte le Québec tôt pour se diriger vers Paris, où il affiche d'emblée une identité nord-américaine affirmée et positive. Il s'éloigne ainsi de *l'image dominante de l'artiste postromantique et 
existentialiste pour adopter plutôt l'attitude type de l'artiste américain moderne, celle du pionnier "super-primitif", viril, impulsif et autodestructeur • (p. 288) qui n'est pas sans rappeler celle de Jackson Pollock. D'ailleurs, pour un temps, leurs pratiques artistiques composées de * dipping " se ressemblent beaucoup. Puis, Riopelle opte pour une pratique picturale rappelant la mosaïque et constituée d'une multitude d'aplats de peinture appliqués à la spatule. C'est à cette époque qu'il s'approprie l'image du trappeur, d'abord soulignée par André Breton en 1949. Selon l'auteure, cette image apparaît principalement dans les entrevues données par l'artiste durant lesquelles il adopte littéralement les stratégies propres au chasseur. Aussi individualiste et nomade qu'un coureur des bois, Riopelle incarne une figure négative et menaçante dans le contexte de la survivance culturelle canadienne-française. Par la suite, il passe de cette identité relativement a authentique * à "l'attitude inverse de l'imposteur, affichant une identité multiple et versatile. (p. 333). Vigneault le compare à la figure du "trickster. Ces changements dans l'attitude de Riopelle, Vigneault les infère de ses techniques représentatives du "low art . comme le bricolage, le graffiti, le pochoir, la peinture en aérosol qui caractérise sa dernière période. Celles-ci, parce qu'issues de la culture populaire, accomplissent une véritable critique de l'élite artistique québécoise.

Louise Vigneault accomplit avec rigueur la tâche de jeter un regard neuf sur l'identité québécoise à travers les trois figures artistiques de Borduas, Sullivan et Riopelle. J'emploie l'expression de a figure artistique - puisque l'angle d'analyse choisi par l'auteure se rapporte davantage aux écrits de ces artistes qu'à leurs pratiques artistiques, ce choix particulièrement manifeste dans le cas de Borduas. À l'exception de l'Hommage à Rosa Luxemburg de Riopelle et, dans une moindre mesure, de la chorégraphie Rose Latulipe de Sullivan, l'auteure ne fait aucune analyse substantielle d'œuvre. L'approche de la production artistique de Borduas, Sullivan et Riopelle demeure le plus souvent à l'étape de la description stylistique et thématique. Cependant, les écrits de ces derniers sont rigoureusement analysés et leurs mises en contexte s'avèrent bien documentées, éclairantes et novatrices. Par ailleurs, cette étude ouvre un domaine nouveau et significatif concernant l'identité québécoise, que l'histoire de l'art québécois a tout intérêt à explorer.

Julie Lavigne

Université McGill 